\title{
Near-IR wide-field-of-view Huygens metalens for outdoor imaging applications
}

Engelberg, Jacob; Zhou, Chen; Mazurski, Noa; Bar-David, Jonathan; Kristensen, Anders; Levy, Uriel

Published in:

Nanophotonics

Link to article, DOI:

10.1515/nanoph-2019-0177

Publication date:

2020

Document Version

Publisher's PDF, also known as Version of record

Link back to DTU Orbit

Citation (APA):

Engelberg, J., Zhou, C., Mazurski, N., Bar-David, J., Kristensen, A., \& Levy, U. (2020). Near-IR wide-field-ofview Huygens metalens for outdoor imaging applications. Nanophotonics, 9(2), 361-370.

https://doi.org/10.1515/nanoph-2019-0177

\section{General rights}

Copyright and moral rights for the publications made accessible in the public portal are retained by the authors and/or other copyright owners and it is a condition of accessing publications that users recognise and abide by the legal requirements associated with these rights.

- Users may download and print one copy of any publication from the public portal for the purpose of private study or research.

- You may not further distribute the material or use it for any profit-making activity or commercial gain

- You may freely distribute the URL identifying the publication in the public portal 


\section{Research article}

\section{Jacob Engelberg, Chen Zhou, Noa Mazurski, Jonathan Bar-David, Anders Kristensen} and Uriel Levy*

\section{Near-IR wide-field-of-view Huygens metalens for outdoor imaging applications}

https://doi.org/10.1515/nanoph-2019-0177

Received June 14, 2019; revised July 24, 2019; accepted July 24, 2019

Abstract: The ongoing effort to implement compact and cheap optical systems is the main driving force for the recent flourishing research in the field of optical metalenses. Metalenses are a type of metasurface, used for focusing and imaging applications, and are implemented based on the nanopatterning of an optical surface. The challenge faced by metalens research is to reach high levels of performance using simple fabrication methods suitable for mass production. In this paper, we present a Huygens nanoantenna-based metalens, designed for outdoor photographic/surveillance applications in the near infrared. We show that good imaging quality can be obtained over a field of view as large as $\pm 15^{\circ}$. This first successful implementation of metalenses for outdoor imaging applications is expected to provide insight and inspiration for future metalens imaging applications.

Keywords: metalens; metasurface; diffractive lens; imaging; optical testing.

\section{Introduction}

The topic of metasurfaces in general, and the specific case of metalenses, is an ongoing area of research [1-4]. The

*Corresponding author: Uriel Levy, Department of Applied Physics, The Faculty of Science, The Center for Nanoscience and Nanotechnology, The Hebrew University Jerusalem Israel, Jerusalem 91904, Israel, e-mail: ulevy@mail.huji.ac.il

Jacob Engelberg, Noa Mazurski and Jonathan Bar-David: Department of Applied Physics, The Faculty of Science, The Center for Nanoscience and Nanotechnology, The Hebrew University Jerusalem Israel, Jerusalem 91904, Israel. https://orcid.org/00000002-7421-914X (J. Engelberg)

Chen Zhou: Department of Micro- and Nanotechnology, Technical University of Denmark, DK-2800 Kongens Lyngby, Denmark Anders Kristensen: Department of Micro- and Nanotechnology, Technical University of Denmark, DK-2800 Kongens Lyngby, Denmark; and Department of Health Technology, Technical University of Denmark, DK-2800 Kongens Lyngby, Denmark main aspirations for metalenses are miniaturization and cost reduction of optical systems by replacement of conventional lenses with metalenses.

Most metalenses operate as a first-order diffractive lens, i.e. the lens introduces a phase function modulo $2 \pi$ to the wavefront, that ideally converts one spherical wavefront (emanating from a point source) to another (by focusing down to a diffraction limited spot). The difference between a metalens and the more traditional surface relief diffractive lens [5] is that in a surface relief diffractive lens, the phase is introduced via an optical path difference resulting from the physical profile of the substrate, while in a metalens the phase is affected via nanostructures patterned on the substrate, which introduce phase delay. Three common methods for introducing the phase delay in dielectric metasurfaces are truncated waveguides [6], geometrical phase [7], and Huygens nanoantennas [8], each having their pros and cons.

Truncated waveguide metasurfaces are composed of nanorods, whose diameters determine the effective refractive index of the optical mode propagating through them, thus allowing phase control. Geometric-phase metasurfaces consist of nanofins, whose orientation determines the phase shift imparted to the light. Huygens metasurfaces consist of resonant structures, e.g. nanodisks. The nanodisks are designed so that the electric and magnetic dipole resonances overlap (thus meeting what is known as the first Kerker condition, in which reflection is inhibited by destructive interference), which results in excellent transmission and a $2 \pi$ phase shift range. The phase shift can be controlled by changing the diameter of the disk [1].

Huygens metalenses have been demonstrated in the microwave spectral region $[9,10]$ and recently also in the optical spectral region [11, 12]. These optical-region metalenses are designed for monochromatic operation, high numerical aperture (NA), and narrow field of view (FOV). Wide-FOV truncated waveguide and geometric-phasetype metalenses have also been demonstrated $[13,14]$.

In this paper, we explore the option of a wide-FOV Huygens-type metalens. The previously demonstrated wide-FOV metalenses have a short focal length $(<1 \mathrm{~mm})$. In our design, we used a longer focal length of $3.36 \mathrm{~mm}$, 
which is more suitable for outdoor imaging, as it provides higher angular resolution necessary for recognition of facial features at a distance of several meters (the focal length of common cell phone camera lenses is about $4 \mathrm{~mm}$ [15]). Our metalens supports a moderately wide FOV of $\pm 15^{\circ}$ over a relatively broad spectral range of up to $\sim 40 \mathrm{~nm}$. Our findings confirm our previously published expectation that good-quality outdoor imaging can be achieved with a non-chromatically corrected metalens, by judiciously choosing the aperture and spectral range [16]. A schematic illustration of the metalens and its application for outdoor imaging is shown in Figure 1A.

\section{Design}

The optical design of our metalens was made using commercial optical design software (Zemax OpticStudio,
Zemax LLC, Kirkland, WA, USA), combined with full-wave finite-difference-time-domain (FDTD) simulations to determine the amplitude and phase response of the nanoantennas. The design concept is based on an aperture stop located at the front focal plane of the lens, which results in a telecentric design (the chief ray exits parallel to optical axis), with good off-axis aberration correction $[13,17]$. The layout of the optical system is shown in Figure 1B. The front aperture is $1.35 \mathrm{~mm}$ in diameter, and the focal length of the metalens is $3.36 \mathrm{~mm}$ (F-number 2.5). The design supports field angles of up to $40^{\circ}$, with near-diffractionlimited performance, using a quadratic diffractive phase function, as described by Eq. (1):

$$
\phi(r)=a r^{2}, a=-1098.2 \mathrm{~mm}^{-2} .
$$

The main performance criterion for imaging lenses is the modulation transfer function (MTF), which describes the resolution of the lens. The MTF gives the modulation

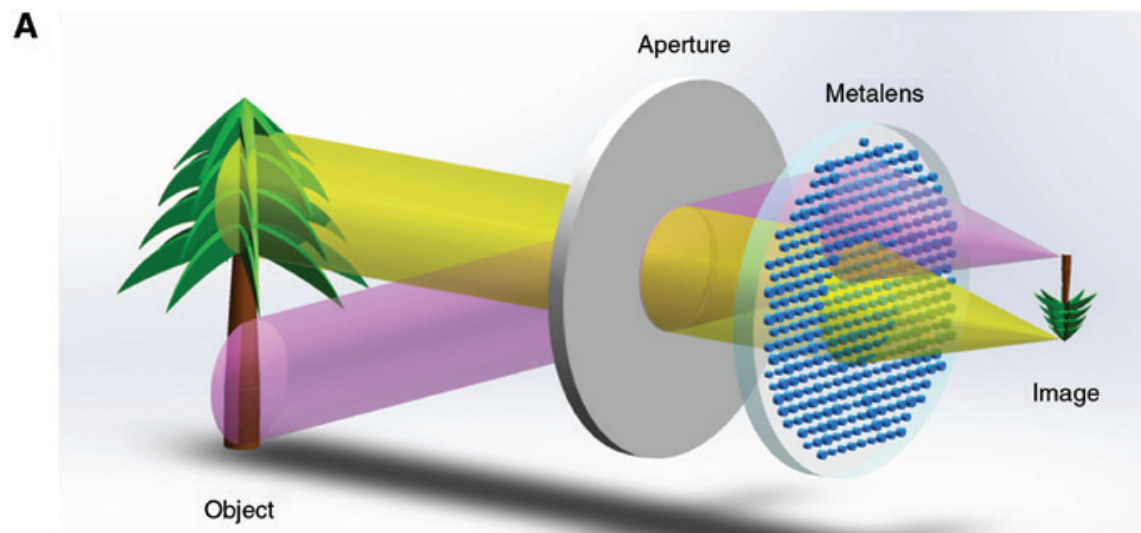

B
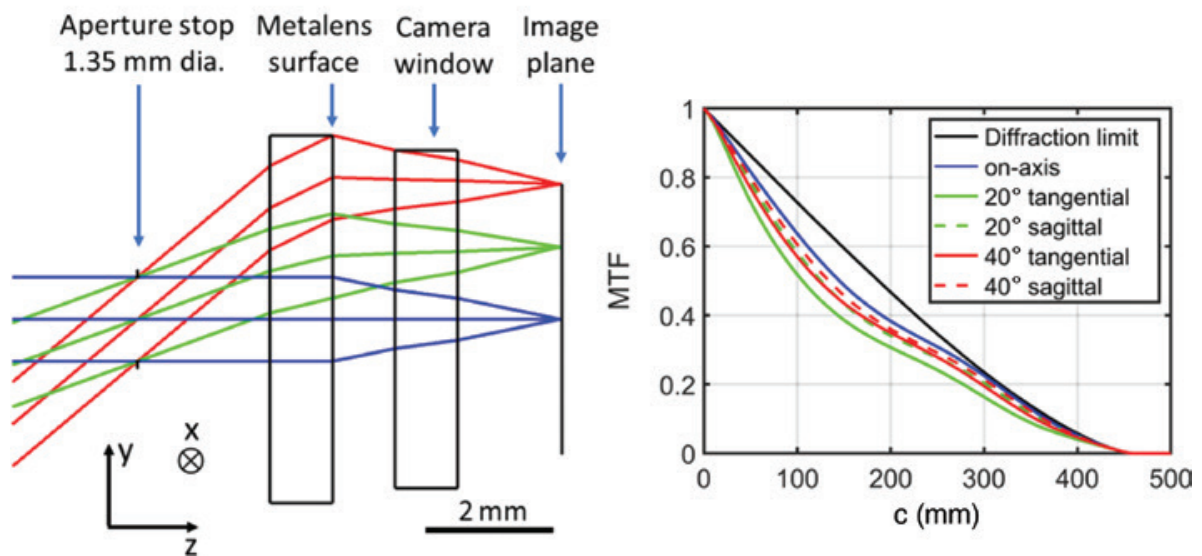

Figure 1: Metalens optical layout and performance.

(A) Schematic illustration of imaging with Huygens metalens. (B) Optical layout. Ray fans drawn are for $0^{\circ}, 20^{\circ}$, and $40^{\circ}$ incidence.

(C) Nominal MTF for monochromatic 850-nm illumination. 
(i.e. contrast) attenuation factor for each spatial frequency, as a result of the lens blur spot. The function describing the blur spot is called the point spread function (PSF) and is a two-dimensional function of the transverse horizontal $(x)$ and vertical $(y)$ image plane coordinates. Integration of the PSF in the horizontal and vertical directions yields the vertical and horizontal one-dimensional line spread functions (LSFs), respectively. The MTF is the Fourier transform of the LSFs; thus, we have two MTFs: horizontal and vertical [18].

The MTF of the nominal metalens design, calculated in Zemax for monochromatic illumination at $850 \mathrm{~nm}$, is shown in Figure 1C. For the on-axis image point, the nominal horizontal and vertical MTFs are identical because of symmetry; however, for the off-axis points, there are two MTF graphs. The direction of the off-axis excursion is called the tangential direction. In our case, this is defined as the $y$-axis direction, as denoted in Figure 1B. The perpendicular direction is called the sagittal direction, which is the $x$-axis direction in our case.

If higher-order diffractive coefficients were used (beyond quadratic), the spherical aberration could be perfectly corrected, so that the on-axis monochromatic MTF (blue line in Figure 1C) would coincide with the diffraction limit. However, when considering the entire FOV, the overall improvement is negligible, which is why we elected to remain with the parabolic phase. Furthermore, previous wide-FOV metalenses used a two-element design [13, 14], with the front element functioning as a Schmidt spherical aberration corrector [19]. This was necessary as they were operating at approximately $F / 1$. However, as our metalens operates at a more moderate aperture of $\mathrm{F} / 2.5$, which is in line with common cellphone camera lenses [15], the additional metasurface is not required, making the implementation of the imaging system much simpler.

In addition to MTF, it is also critical for an imaging lens to provide illumination that is both strong enough and reasonably uniform over the FOV. As our lens is designed to operate with broad-spectrum illumination, we must allow a sufficiently wide spectral range of light to pass through to obtain a good illumination level. Unfortunately, the wider the spectral range, the larger the chromatic blurring will be, resulting in a degradation of the monochromatic MTF shown in Figure $1 \mathrm{C}$ to the polychromatic MTFs that will be shown later (Figure $4 \mathrm{~A}-\mathrm{C}$ ). This trade-off between resolution and illumination signal was explored from a theoretical point of view in our previous paper [16]. The results shown in this paper confirm our previous expectation that at outdoor illumination levels, one can obtain good signal and resolution by using moderate spectral bands of up to $40 \mathrm{~nm}$.
An additional performance parameter, not accounted for by MTF, is the geometrical distortion, which is a distortion of the shape of the imaged objects, without affecting the image resolution. The relative distortion is defined as the ratio between the shift in position of an image point relative to the absolute ideal position. Our telecentric type of design exhibits negative ("barrel") distortion, reaching $23 \%$ at $40^{\circ} \mathrm{FOV}$, but only $3.4 \%$ at $15^{\circ} \mathrm{FOV}$, as shown in Figure S1A. As a rule of thumb, a distortion of up to $10 \%$ is not disturbing to a standard viewer. Distortion can also be corrected using established image processing techniques [20].

To reduce the fabrication effort, the lens aperture diameter was limited to $2 \mathrm{~mm}$. This resulted in blocking of some rays (which optical designers call "vignetting”) at off-axis incidence angles $>8^{\circ}$, causing a gradual drop-off in illumination as shown in Figure S1B. However, at incidence angles up to $15^{\circ}$, we still have $>65 \%$ relative illumination (this is without considering the Huygens antenna response to different incidence angles, which will be discussed in Section 3.2).

To implement the phase shifts required for the diffractive phase function, while maintaining high transmission, we used Huygens nanoantennas. The nanoantenna simulation was performed using a commercial threedimensional FDTD code (Lumerical Inc., Vancouver, BC, Canada). The antennas are made of amorphous silicon on a glass substrate and are covered with a thin layer of polymethylmethacrylate (PMMA) ( $\sim 300 \mathrm{~nm}$ thick). The lattice period was chosen to be $500 \mathrm{~nm}$. This period was chosen considering sub-wavelength and phase sampling requirements[21] (see details in section 2 of the Supplementary Material), in addition to antenna coupling, which occurs at smaller periods, and interferes with achieving the Kerker condition [22].

To find the optimal antenna dimensions, we performed a numerical scan over the antenna radius and height, while monitoring the transmission and phase of a periodic antenna array. It turned out that for a hexagonal lattice with a period of $500 \mathrm{~nm}$, the optimal antenna height (for which the electric and magnetic dipole resonances overlap at a wavelength of $850 \mathrm{~nm}$ ) is $140 \mathrm{~nm}$. The nominal transmission and phase response for a periodic nanoantenna array is shown in Figure 2. Field plots showing the electric and magnetic dipole resonances are presented in section 3 of the Supplementary Material.

To implement the desired quadratic phase function, eight discrete antenna radii were chosen, spanning a range of $100-164 \mathrm{~nm}$, such that the phase shifts are equally spaced over the $2 \pi$ range. It can be seen from Figure $2 \mathrm{C}$ 
A
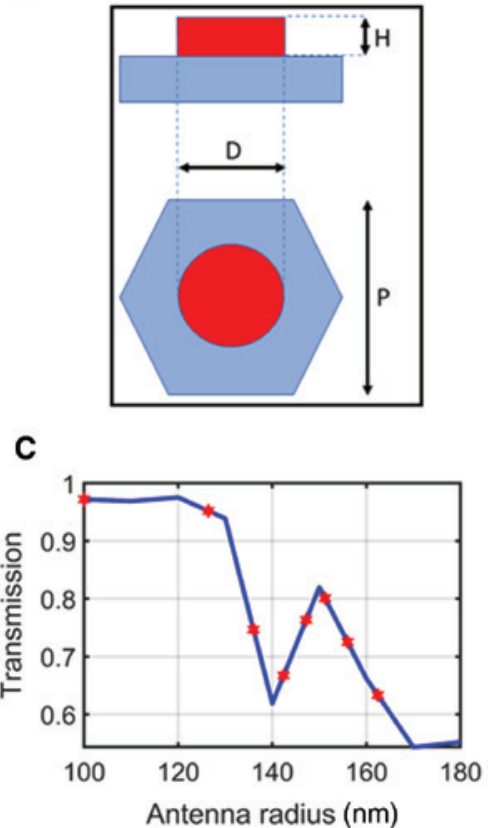

B

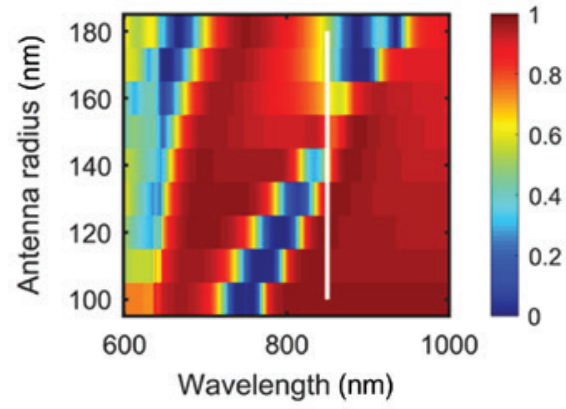

D

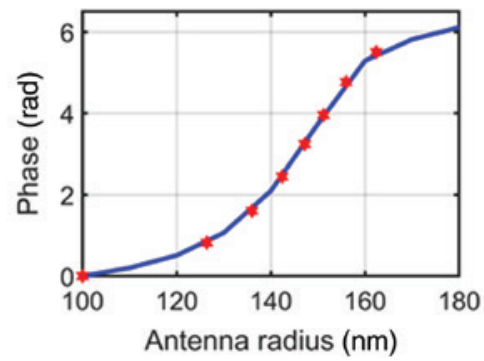

Figure 2: Response of Huygens a-Si antenna array on glass.

(A) Nano-antenna unit cell design. $P=500 \mathrm{~nm}, H=140 \mathrm{~nm}$, and $D$ is in the range of $200-328 \mathrm{~nm}$. (B) Transmission as a function of wavelength and antenna radius. White vertical line is the section along which graphs $C$ and $D$ are drawn. (C) Transmission at $850 \mathrm{~nm}$ as a function of antenna radius. (D) Phase at $850 \mathrm{~nm}$ as a function of antenna radius. Red markers are at the location of the eight antenna radii used in our metalens.

that transmission $>60 \%$ is maintained for the full range of radii. The parabolic phase function extracted from the above-mentioned Zemax design was used to determine which of the eight antenna radii should be placed at each transverse location across the lens aperture. The metalens graphic layout together with optical and scanning electron microscopy (SEM) images of the manufactured metalens are shown in Figure 3.

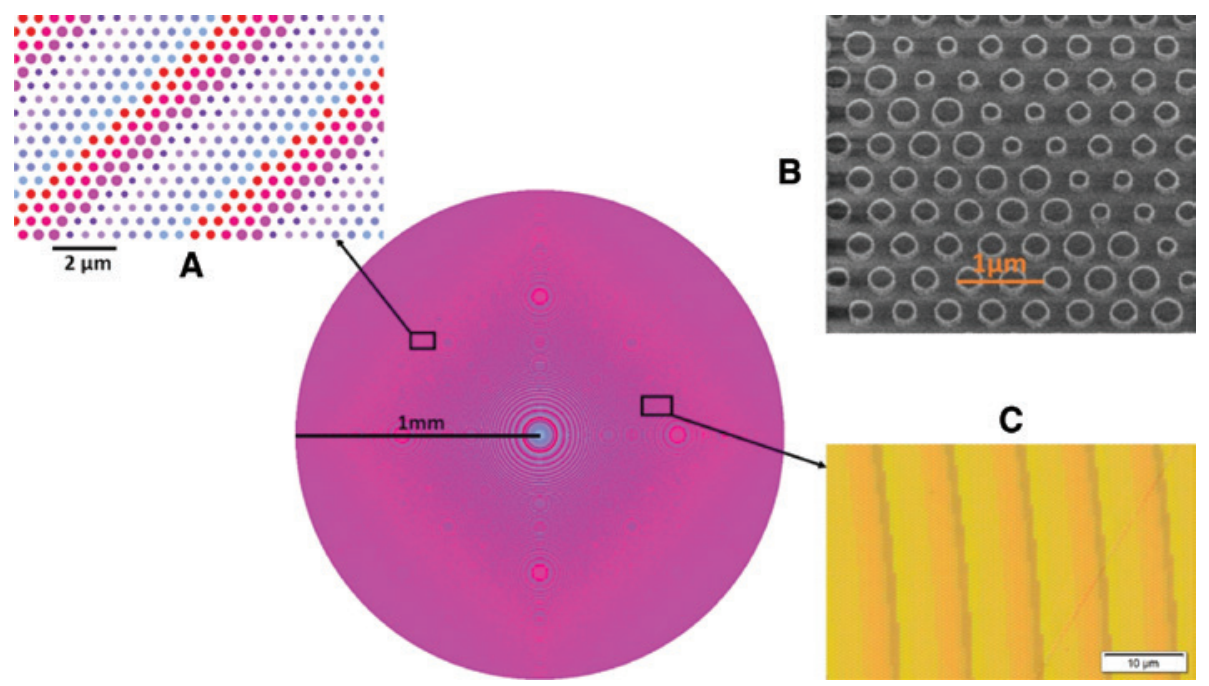

Figure 3: Metalens design pattern for e-beam lithography.

Each color represents a different antenna radius. The bulls-eye pattern in the center of the metalens are the Fresnel zones. Other bulls-eye patterns are aliasing artifacts. Insets: (A) Zoom-in on antenna pattern. (B) SEM image of metalens section. (C) Optical microscope image of metalens section. 


\section{Results}

\subsection{Resolution}

The MTF of the lens was measured on-axis and off-axis at several spectral widths, with unpolarized light, using the setup described in Section 5. The measurement results are shown in Figure 4, in comparison to the simulated results. The MTF simulation results are affected most by axial and lateral chromatic aberration (a detailed discussion of the chromatic aberrations and their influence on MTF can be found in our previous theoretical paper [16]). The spectral weights used in our simulation are based on the measured spectrum of the light source used in our test setup with different spectral filters, and spectral responsivity of the camera. The spectral contributions of the setup optics and of the metalens itself (which is the first-order metalens efficiency shown later in Figure 5A) were neglected, as they are rather flat over the relevant spectral range.

By comparing Figure 4A and D, we can see an excellent match between the measured and simulated results on-axis. Note that there is only one on-axis simulated graph for each spectral width in Figure 4A, because of symmetry, while for the measured results of Figure 4D there are two graphs, as tolerances and noise break the symmetry. The measured result for 2.5-nm spectral width is slightly lower than the simulation. This is most likely

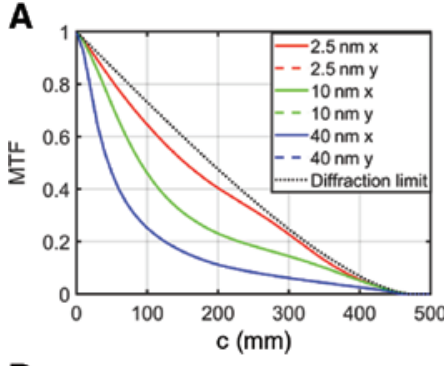

D

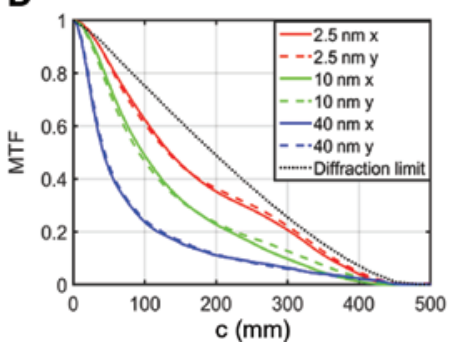

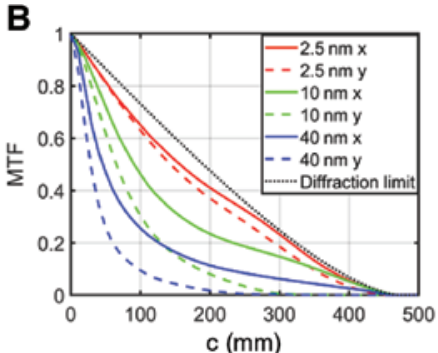

E

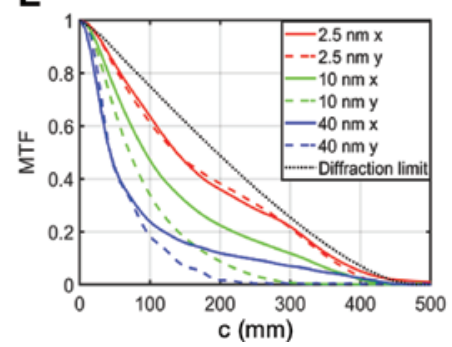

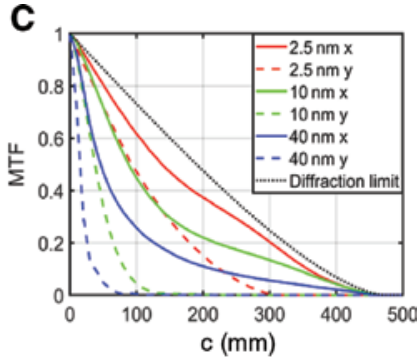

$\mathbf{F}$

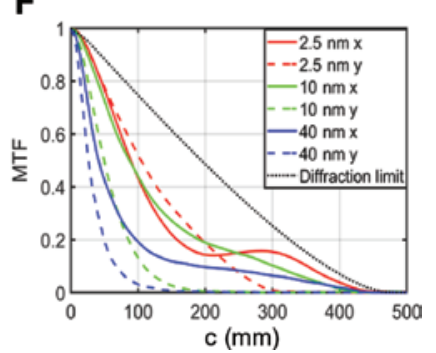

Figure 4: Polychromatic MTF measured vs. simulated results.

(A, B, and C) Simulated results for on-axis, $0.4-\mathrm{mm}$ off-axis, and $0.8-\mathrm{mm}$ off-axis (in the image plane), respectively. (D, E, and F) Corresponding measured results. In each graph, results for three spectral widths $(2.5,10$, and $40 \mathrm{~nm})$ are presented and compared to the theoretical diffraction limit. The $y$-axis is the tangential direction (the direction in which we went off-axis).
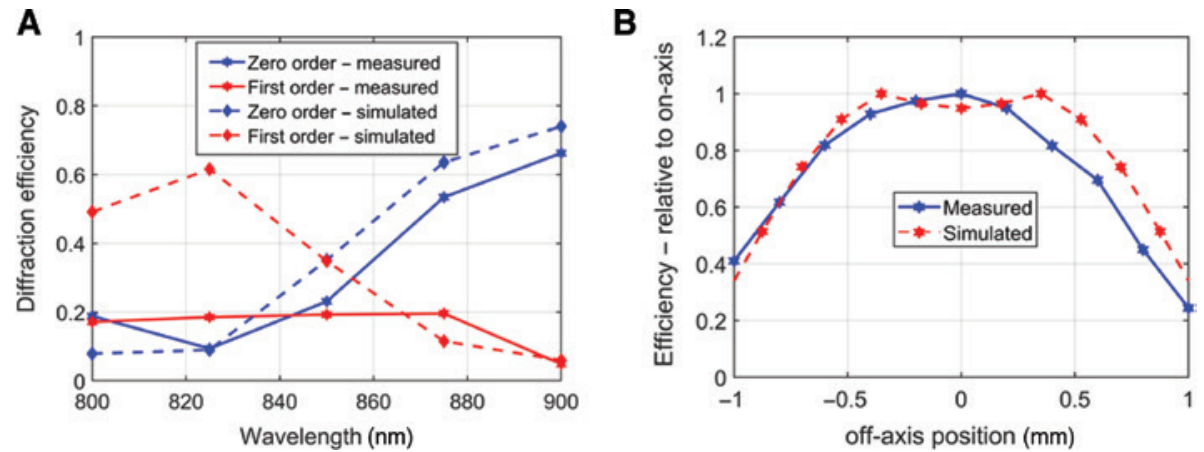

Figure 5: Metalens diffraction efficiency.

Measured results compared to simulation. (A) On-axis, first order, and zero order. (B) Off-axis, first order. A distance of 1-mm off-axis in the image plane corresponds to a field angle of 17.3 degrees. 
caused by the inaccuracy in centration of the stop relative to the metalens, which gives rise to coma aberration on-axis. For the off-axis, comparing Figure 4B vs. E and $\mathrm{C}$ vs. F, we see a good match between theory and experiment for the $y$ (tangential) axis. Interestingly, the measured results are slightly better than the simulated ones, probably because we clipped the distant edges of the PSF during measurement. In the $x$-axis (sagittal) direction, the measured results match the theory very well, except for the 2.5-nm spectral width. Here, the measured results are significantly worse than expected, again most likely due to the decentration tolerance between the stop and the metalens, which is more sensitive off-axis than on-axis. The effect of vignetting is felt at the 0.8 -mm off-axis point with 2.5-nm spectral width, in the $y$-direction (red dashed line in Figure 4C,F). In this case, the performance is diffraction limited; however, the diffraction limit is lower, with a cutoff at about $300 \mathrm{c} / \mathrm{mm}$ instead of $450 \mathrm{c} / \mathrm{mm}$.

\subsection{Efficiency}

Next, we measured the metalens efficiency, i.e. what percent of the incident light gets to the first-order focal point? The measurement was performed using the same setup used to measure MTF, at several wavelengths (for details, see Section 5). The results are shown in Figure 5 and are compared to expected diffraction efficiency values based on simulation. The simulation was performed using Matlab software (Mathworks Inc., Natick, MA, USA), while incorporating the transmission and phase values that were calculated by FDTD for each of the eight radii of antennas. For the off-axis points, a beam incident at an angle was used in the FDTD simulation, together with Bloch boundary conditions, at 850-nm wavelength. The efficiency measurement was performed with unpolarized light. To compare measured results to simulations at non-normal incidence angles, we averaged the simulation results for transverse electric (TE) and transverse magnetic (TM) polarization.

To simplify the scalar diffraction Matlab simulation, we did not use the lens itself for the diffraction efficiency calculation, but rather a blazed grating. The efficiencies in both cases are similar, as the lens can be viewed locally as a grating with radially varying period [23]. In our FDTD simulations, we used the nominal antenna radii. As to the accuracy of our SEM measurement, the radii came out close to nominal. In the simulations, we used an antenna height value of $135 \mathrm{~nm}$, which is the value measured by surface profilometry, rather than the nominal $140 \mathrm{~nm}$.

In the simulation results shown in Figure 5A, a shift of the maximum efficiency wavelength from the nominal design value of $850 \mathrm{~nm}$ to a measured value of $825 \mathrm{~nm}$ is observed (peak of red dashed line). This shift is the result of the deviation in antenna height from 140 to $135 \mathrm{~nm}$, as mentioned above. The measured first-order diffraction efficiency is much lower than the simulated values, reaching a maximum efficiency of about $20 \%$ as compared with the simulated value of $60 \%$. Indeed, the simulation is expected to be slightly optimistic, as we did not account for the reduced number of phase steps near the edge of the metalens, and for the spatial phase sampling errors that become significant at the smaller zone widths. However, the contribution of these effects is not expected to be very significant, as the minimal diffractive period of the metalens is $2.86 \mu \mathrm{m}$, still supporting more than five phase samples in the smallest period [24]. There is about $8 \%$ of power loss due to Fresnel reflections from the two sides of the element (substrate side and PMMA cover side). An important possible contributor is the difference between the FDTD simulations and the experimental results regarding the interaction between antennas. The simulation assumes a periodic structure, whereas in the metalens neighboring antennas have different radii. Still, we anticipate that the reduced efficiency is caused mostly by errors in the antenna phase response resulting from manufacturing tolerances (errors in disk circularity, diameter, height, verticality of sidewalls, etc.), so the fundamental limit is probably significantly better than what we achieved in this first attempt. This is a subject for further investigation.

The efficiency of our metalens is described by the first-order diffraction efficiency shown in Figure 5A. Light going to zero or higher diffraction orders (positive or negative) will contribute to uniform background light in the image, reducing the overall image contrast, but not affecting the limiting resolution. Only the zero-order diffraction efficiency is shown in the figure, as it is the dominant contribution.

The off-axis efficiency simulation, shown in Figure 5B, accounts for vignetting (caused by the 2-mm metalens diameter), which we will call "geometrical efficiency", and also for the variation in diffraction efficiency caused by the change in antenna response (transmission and phase) as a function of the incidence angle. There is a good match between measured and simulated results. The dip in simulated efficiency at normal incidence stems from using the experimentally observed height of $135 \mathrm{~nm}$ rather than the nominal $140 \mathrm{~nm}$. This causes optimum efficiency to be obtained slightly off-axis. This effect is not evident in the measured results, probably because of manufacturing tolerances. In addition, the measured efficiency is slightly asymmetric with respect to the off-axis position (shifted to the left). This is probably because of the slight mechanical 
decenter of the iris with respect to the metalens, resulting in asymmetric vignetting.

\subsection{Outdoor imaging}

To obtain a qualitative assessment of the imaging quality of the metalens, we adapted it to a digital monochrome video camera (DCC1545M from Thorlabs Inc., Newton, NJ, USA). In Figure 6, an outdoor picture of our Israeli research group, taken with the metalens, is shown. The scene was illuminated by natural sunlight, with a band-pass filter (Thorlabs FB800-10, central wavelength $800 \mathrm{~nm}, 10-\mathrm{nm}$ full-width at half-maximum) incorporated in front of the metalens. No post-processing was performed on the image, except for cropping of the dark areas in the periphery. To our knowledge, this is the first time that a metalens has been used for imaging in an outdoor environment.

Most previous metalens testing was performed by viewing the image with an auxiliary microscope system. In one case, the metalens was coupled directly to a camera [13], but testing was only performed in the laboratory. In our experience, it important to perform field testing of an imaging system in addition to laboratory testing. The main reason for this is that the illumination conditions that exist in the field are different from those in the laboratory. (Other reasons include atmospheric effects, temperature gradients, mechanical stability issues, etc.
These are usually more significant for long-focal-length systems, and are therefore less relevant for our metalens.)

The importance of field testing of a metalens is exemplified by an image artifact that we discovered while performing indoor imaging with ambient light. The image is shown in Figure S9 (left). As can be seen, there is a bright circle with jagged edges in the center of the image (this is aside from the larger circle that limits the FOV and is a result of the drop-off in illumination as shown in Figure 5B). The observation of these jagged edges is a clear indication that what we are seeing is an image of our mechanical iris, whose machined edges are jagged (this was verified by rotating the iris, which caused the jagged edges in the image to rotate as well).

Such a ghost image of the iris is known as a "flare spot" and is a well-known phenomenon in photography, as shown in Figure S9 (right). It generally arises from a double reflection in the system. Yet, our setup consists of a single optical element only, with plane surfaces, so it is impossible for the iris to be imaged on the sensor by double reflection. Where is the ghost image coming from? Simple geometrical optics considerations, supported by Zemax simulation, shown in Figure S10, indicate that it is coming from the second order of diffraction. For the second order of diffraction, the focal length of the metalens is half the nominal focal length. As the iris is located at the nominal front focal plane of the lens, and the sensor at the rear focal plane, in the second order of diffraction

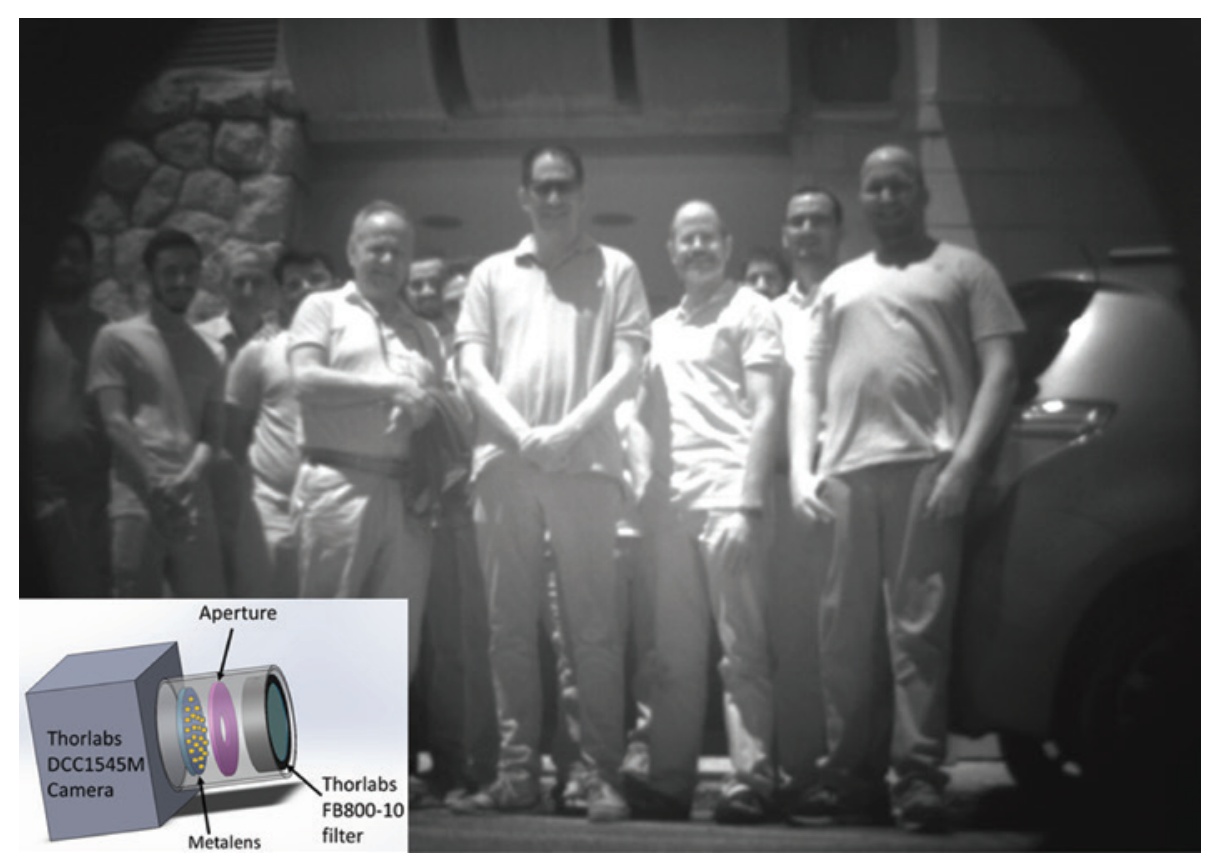

Figure 6: Metalens outdoor image.

NanoOpto group members in Hebrew University. Inset: imaging setup. 
the iris is imaged onto the sensor in a $2 f-2 f$ configuration (i.e. a magnification of 1). Such an effect exists to some extent in all wide-FOV telecentric metalenses due to slight deviations of the fabricated devices from the design target. Additional discussion, including comparison of our metalens to a commercial lens, can be found in section 6 of the Supplementary Material.

\section{Discussion}

The advantage of the Huygens metalens over previously studied truncated waveguide or geometrical phase metalenses $[2,3]$ is the smaller antenna aspect ratio, which simplifies the fabrication process. Based on our design, the antenna height is $140 \mathrm{~nm}$ and the smallest diameter is $200 \mathrm{~nm}$, so the highest aspect ratio is about 0.7. This is about an order of magnitude lower than the aspect ratio needed for the implementation of geometrical phase or truncated waveguide nanoantennas.

Alongside with the high merits of the Huygens-type metalens, the Huygens-type nanoantenna-based metalens is more sensitive to angular, wavelength, and dimensional deviations. This is a result of the fact that the Huygens concept is based on a resonant effect, which naturally has a narrower spectral response as compared to the other, nonresonant approaches. We have found that while this high sensitivity significantly degrades the efficiency of the metalens, it has a marginal effect on the obtainable resolution.

The sensitivity of efficiency to manufacturing tolerances is inherent in the Huygens design and can be overcome by an improved and more accurate fabrication process. The sensitivity of efficiency to wavelength was found to be moderate, allowing for about 40-nm bandwidth to be used, based on Figure 5A (first-order simulated result). In the case of a chromatic lens (i.e. not corrected for chromatic aberration), we will be limited to a moderate bandwidth, based on the resolution-illumination trade-off. An analysis of the optimal bandwidth to use for a given metalens is beyond the scope of this paper. Such a discussion can be found in our previous paper[16]. The sensitivity to the angle of incidence limits the FOV of a standard Huygens metalens to a maximum of about $10-20^{\circ}$, as shown in Figure $5 \mathrm{~B}$. A detailed comparison of the metalens reported in this paper with previously reported wide-FOV metalenses can be found in section 7 of the Supplementary Material.

Current metalens research is focusing on correcting the chromatic aberration of metalenses and providing tunability of the metalens focal length. Chromatic aberration can be corrected by using dispersion-engineered antennas $[25,26]$; however, this works only for a narrow spectral band or low Fresnel number (which is proportional to the NA product) [27]. Other options are transverse $[28,29]$ or longitudinal [30] multiplexing of metalenses. In all cases, the chromatic correction comes at the expense of efficiency and manufacturability. Several methods have been demonstrated for tuning metalens focal length some of them external to the metalens $[12,31]$ and some more integral to the lens [32-34].

Finally, the potential impact of optical metalenses is currently subject to discussion. They are being compared with some pioneering previous works implementing surface relief diffraction lenses. Indeed, what reason is there to choose a metalens-type diffractive lens over a surface relief diffractive lens? In Refs. [35, 36], it is argued that the only advantage of a metasurface over a diffractive surface is if one wants to manipulate the polarization. This would mean that conventional metalenses, which manipulate only the phase of the light, are redundant. Here, we would like to highlight several other potential advantages: (a) The metalens can accomplish the same task as the diffractive lens using a binary structure instead of multilevel or kinoform, so it is easier to manufacture. (b) Metalenses have less shading effect for small diffractive periods [37] (which is very important for high-power lenses that necessarily have small periods near the edge). (c) Metalenses allow dispersion control of the phase, thus allowing control of spectral diffraction efficiency and chromatic aberration [38, 39]. It is further argued that the aspect ratio of the nanoantennas is higher than that of an equivalent diffractive-type lens. While this may be the case for truncated waveguide and geometrical phase-type metalenses (depending on the NA of the lens, as for a diffractive element, the aspect ratio increases for larger NA), the Huygens-type metalens presented in this paper is based on nanoantennas with low aspect ratios. As mentioned above, the highest aspect ratio of our metalens is about 0.7 , whereas the diffractive lenses discussed in Refs. $[35,36]$ report an aspect ratio of about 1. To summarize, in our opinion, surface relief diffractive elements and the various types of metasurface diffractive elements each have their advantages and disadvantages. The choice of which type of diffractive element to use is application specific and depends upon manufacturing capabilities and performance requirements.

\section{Materials and methods}

The MTF simulation shown in Figure $4 \mathrm{~A}-\mathrm{C}$ was performed using Matlab in conjunction with Zemax. The simulation could not be performed using a standard Zemax simulation because of the large number of wavelengths required 
to obtain accurate MTF results for a diffractive lens possessing large chromatic aberration (Zemax supports only up to 24 wavelengths, while we used 85,160 , and 453 wavelengths for spectral widths of $2.5,10$, and $40 \mathrm{~nm}$, respectively). Therefore, a Zemax Programming Language macro was written in Zemax to calculate the optical transfer function (OTF) at each single wavelength and to output the data to a text file. Matlab was then used to sum the OTFs of all wavelengths, with appropriate spectral weighting, to obtain the total OTF. The absolute value of the OTF was then taken to obtain the MTF.

In our previous paper [16], we simulated the MTF of a metalens assuming a top-hat-shaped PSF at each wavelength, resulting from chromatic defocus, which is a geometrical optics approximation. While the geometrical optics approximation is accurate for a large defocus, such that the distance from the focal spot to the image plane can be considered "far-field", for our case this is not accurate, as the chromatic defocus is relatively small. For the simulations in this paper, we therefore used a physical optics calculation based on the Fraunhofer approximation, as performed by the "FFT MTF" simulation in Zemax. We verified that the defocus spots are small enough in our case so that the Fraunhofer approximation is accurate (see section 4 of the Supplementary Material) [40].

Our metalens was fabricated on a 1-mm-thick glass substrate using electron beam lithography. A 135-nmthick layer of amorphous silicon was deposited on the glass using plasma-enhanced chemical vapor deposition (Plasmalab System 100, Oxford Instruments, Yatton, Bristol, UK). The substrate was then spin coated with chemical semi-amplified electron beam resist (CSAR, ALLRESIST GmbH, Strausberg, Germany), and thermal evaporation of a 20-nm-thick aluminum layer for decharging was performed. Following electron beam writing, the CSAR was developed using ALLRESIST AR 600-546 and a 50-nm-thick layer of aluminum was deposited by electron beam evaporation, for use as a hard mask. Lift-off was then performed using ALLRESIST AR 600-71 solvent. Following lift-off, the sample was etched using reactive-ion etching (Corial 200I ICP RIE, CORIAL, Bernin, France), and the aluminum mask was removed using an aluminum etch solvent (80-15-3-2, J.T.Baker, Austin, TX, USA). The sample was then spin coated with a 300-nm-thick layer of PMMA (950 PMMA A2, MicroChem Corp., Westborough, MA, USA) for use as an index matching protective cover.

MTF and efficiency were measured using the experimental setup shown in Figure S2. The fiber-coupled light source is used as an input to the collimator, to produce a high-quality collimated beam. The collimated beam is used as the input to the metalens. A mechanical stop is placed in front of the metalens, at the nominal stop position. Note that the mechanical stop is centered with respect to the metalens; however, when measuring offaxis, the beam wanders to the side of the metalens, since the stop is located at the front focal plane of the metalens. The metalens focuses the light to a point, the intensity profile of which is our PSF. The PSF is then measured using a $50 \times$ microscope.

The efficiency was measured by integrating the camera pixel values for several situations, shown in Figure S3: (a) same as MTF measurement - gives the power in first diffraction order; (b) $50 \times$ objective removed - gives the power in the zero order; and (c) metalens removed but aperture stop still in place - gives the total power, which is our reference signal.

Funding: This study was funded by the Israel Ministry of Science, Technology and Space.

\section{References}

[1] Decker M, Staude I, Falkner M, et al. High-efficiency dielectric Huygens' surfaces. Adv Opt Mater 2015;3:813-20.

[2] Arbabi A, Horie Y, Ball AJ, Bagheri M, Faraon A. Subwavelengththick lenses with high numerical apertures and large efficiency based on high-contrast transmitarrays. Nat Commun 2015;6:7069.

[3] Khorasaninejad M, Chen WT, Devlin RC, Oh J, Zhu AY, Capasso F. Metalenses at visible wavelengths: diffraction-limited focusing and subwavelength resolution imaging. Science 2016;352: 1190-4.

[4] Hasman E, Kleiner V, Biener G, Niv A. Polarization dependent focusing lens by use of quantized Pancharatnam-Berry phase diffractive optics. Appl Phys Lett 2003;82:328-30.

[5] O'Shea DD, Suleski TJ, Kathman AD, Praather DW. Diffractive optics. Bellingham, WA, USA, SPIE Press, 2003.

[6] Lalanne P. Waveguiding in blazed-binary diffractive elements. J Opt Soc Am A 1999;16:2517-20.

[7] Devlin RC, Khorasaninejad M, Chen WT, Oh J, Capasso F. Broadband high-efficiency dielectric metasurfaces for the visible spectrum. Proc Natl Acad Sci 2016;113:10473-8.

[8] Chong KE, Staude I, James A, et al. Polarization-independent silicon metadevices for efficient optical wavefront control. Nano Lett 2015;15:5369-74.

[9] Bin Wang Z, Bin Wang Z, Feng YJ, Chen ZN. An ultrathin microwave Huygens' metasurface lens. IEEE APCAP Proc 2015; 227-8.

[10] Chen K, Feng Y, Monticone F, et al. A reconfigurable active Huygens' metalens. Adv Mater 2017;29:1606422.

[11] Paniagua-Domínguez R, Yu YF, Khaidarov E, et al. A metalens with a near-unity numerical aperture. Nano Lett 2018;18: 2124-32.

[12] Afridi A, Canet-ferrer J, Philippet L, Osmond J, Berto P, Quidant R. Electrically varifocal silicon metalens. ACS Photonics 2018;5:4497-503. 
[13] Arbabi A, Arbabi E, Kamali SM, Horie Y, Han S, Faraon A. Miniature optical planar camera based on a wide-angle metasurface doublet corrected for monochromatic aberrations. Nat Commun 2016;7:13682.

[14] Groever B, Chen WT, Capasso F. Meta-lens doublet in the visible region. Nano Lett 2017;17:4902-7.

[15] Liu YT. Review and design a mobile phone camera lens for 21.4 megapixels image sensor, Master's thesis. University of Arizona, 2017.

[16] Engelberg J, Levy U. Optimizing the spectral range of diffractive metalenses for polychromatic imaging applications. Opt Express 2017;25:21637-51.

[17] Buralli DA, Morris GM. Design of a wide field diffractive landscape lens. Appl Opt 1989;28:3950-9.

[18] Goodman JW. Introduction to Fourier optics, 2nd ed. New York, NY, USA, McGraw-Hill, 1996.

[19] Smith WJ. Modern lens design. McGraw-Hill, 1992.

[20] Van der Jeught S, Buytaert JAN, Dirckx JJJ. Real-time geometric lens distortion correction using a graphics processing unit. Opt Eng 2012;51:027002.

[21] Chen WT, Zhu AY, Khorasaninejad M, Shi Z, Sanjeev V, Capasso F. Immersion meta-lenses at visible wavelengths for nanoscale imaging. Nano Lett 2017;17:3188-94.

[22] Kuznetsov Al, Miroshnichenko AE, Brongersma ML, Kivshar YS, Luk'yanchuk B. Optically resonant dielectric nanostructures. Science 2016;354:aag2472.

[23] Herzig HP, editor. Micro-optics. London, UK, Taylor \& Francis, 1997.

[24] Levy U, Mendlovic D, Marom E. Efficiency analysis of diffractive lenses. J Opt Soc Am A 2001;18:86-93.

[25] Aieta F, Kats MA, Genevet P, Capasso F. Multiwavelength achromatic metasurfaces by dispersive phase compensation. Science 2015;347:1342-5.

[26] Wang S, Wu PC, Su VC, et al. A broadband achromatic metalens in the visible. Nat Nanotechnol 2018;13:227-32.

[27] Shrestha S, Overvig AC, Lu M. Broadband achromatic dielectric metalenses. Light Sci Appl 2018;7:85.

[28] Arbabi E, Arbabi A, Kamali SM, Horie Y, Faraon A. Multiwavelength polarization-insensitive lenses based on dielectric metasurfaces with meta-molecules. Optica 2016;3:628-33.
[29] Li K, Guo Y, Pu M, et al. Dispersion controlling meta-lens at visible frequency. Opt Express 2017;25:21419-27.

[30] Avayu O, Almeida E, Prior Y, Ellenbogen T. Composite functional metasurfaces for multispectral achromatic optics. Nat Commun 2017;8:14992.

[31] Arbabi E, Arbabi A, Kamali SM, Horie Y, Faraji-Dana MS, Faraon A. MEMS-tunable dielectric metasurface lens. Nat Commun 2018;9:812.

[32] Kamali SM, Arbabi E, Arbabi A, Horie Y, Faraon A. Highly tunable elastic dielectric metasurface lenses. Laser Photonics Rev 2016;10:1002-8.

[33] She A, Zhang S, Shian S, Clarke DR, Capasso F. Adaptive metalenses with simultaneous electrical control of focal length, astigmatism, and shift. Sci Adv 2018;4:eaap9957.

[34] Iyer PP, DeCrescent RA, Lewi T, Antonellis N, Schuller JA. Uniform thermo-optic tunability of dielectric metalenses. Phys Rev Appl 2018;10:044029.

[35] Wang P, Mohammad N, Menon R. Chromatic-aberration-corrected diffractive lenses for ultra-broadband focusing. Sci Rep 2016;6:21545.

[36] Mohammad N, Meem M, Shen B, Wang P, Menon R. Broadband imaging with one planar diffractive lens. Sci Rep 2018;8:2799.

[37] Lee MSL, Lalanne P, Rodier JC, Chavel P, Cambril E, Chen Y. Imaging with blazed-binary diffractive. J Opt A Pure Appl Opt 2002;4:S119-24.

[38] Aieta F, Kats MA, Genevet P, Khorasaninejad R, Capasso F. Achromatic metasurfaces by dispersive phase compensation. 2015 IEEE Photon Conf IPC 2015;2015:202-3.

[39] Arbabi E, Arbabi A, Kamali SM, Horie Y, Faraon A. Controlling the sign of chromatic dispersion in diffractive optics. Optica 2017;4:625-32.

[40] Engelberg YM, Ruschin S. Fast method for physical optics propagation of high-numerical-aperture beams. JOSA A 2004;21:2135-45.

Supplementary Material: The online version of this article offers supplementary material (https://doi.org/10.1515/nanoph-2019-0177). 